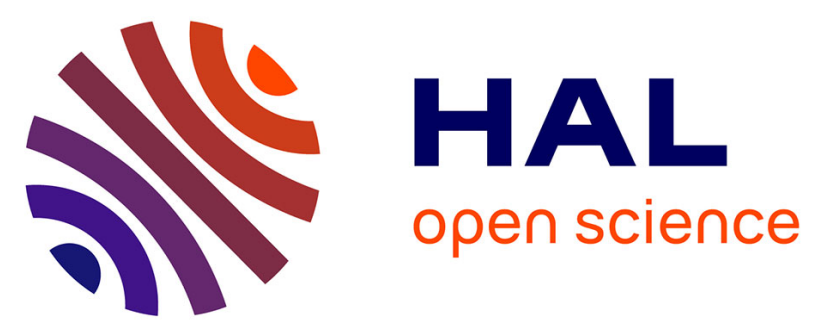

\title{
How motility can enhance mass transfer and absorption in the duodenum: Taking the structure of the villi into account
}

\author{
Yanan Zhang, Peng Wu, Romain Jeantet, Didier Dupont, Guillaume \\ Delaplace, Xiao Dong Chen, Jie Xiao
}

\section{To cite this version:}

Yanan Zhang, Peng Wu, Romain Jeantet, Didier Dupont, Guillaume Delaplace, et al.. How motility can enhance mass transfer and absorption in the duodenum: Taking the structure of the villi into account. Chemical Engineering Science, 2020, 213, pp.115406. 10.1016/j.ces.2019.115406 . hal02862203

\section{HAL Id: hal-02862203 \\ https: / hal.inrae.fr/hal-02862203}

Submitted on 9 Jun 2020

HAL is a multi-disciplinary open access archive for the deposit and dissemination of scientific research documents, whether they are published or not. The documents may come from teaching and research institutions in France or abroad, or from public or private research centers.
L'archive ouverte pluridisciplinaire HAL, est destinée au dépôt et à la diffusion de documents scientifiques de niveau recherche, publiés ou non, émanant des établissements d'enseignement et de recherche français ou étrangers, des laboratoires publics ou privés.

\section{(1) (1) $\$$}

Distributed under a Creative Commons Attribution - NonCommercial - NoDerivatives 44.0 


\section{How motility can enhance mass transfer and absorption in the duodenum: Taking the structure of the villi into account}

\section{H I G H L I G H T S}

- A CFD model for duodenum that explicitly takes the villi structure into account.

- Enhancement of mass transfer and absorption quantified by an enhancement factor.

- Ambient bulk flow reduces the enhancement effect from the pendular activity.

- Top part of villi plays a significant role in nutrient absorption.

\section{A R T I C L E I N F O}

Article history:

Received 3 September 2019

Received in revised form 22 November 2019

Accepted 29 November 2019

Available online 2 December 2019

\section{Keywords:}

Motility

Small intestine

Villi

Mass transfer and absorption

Numerical simulation

Moving mesh method
G R A P H I C A L A B S T R A C T

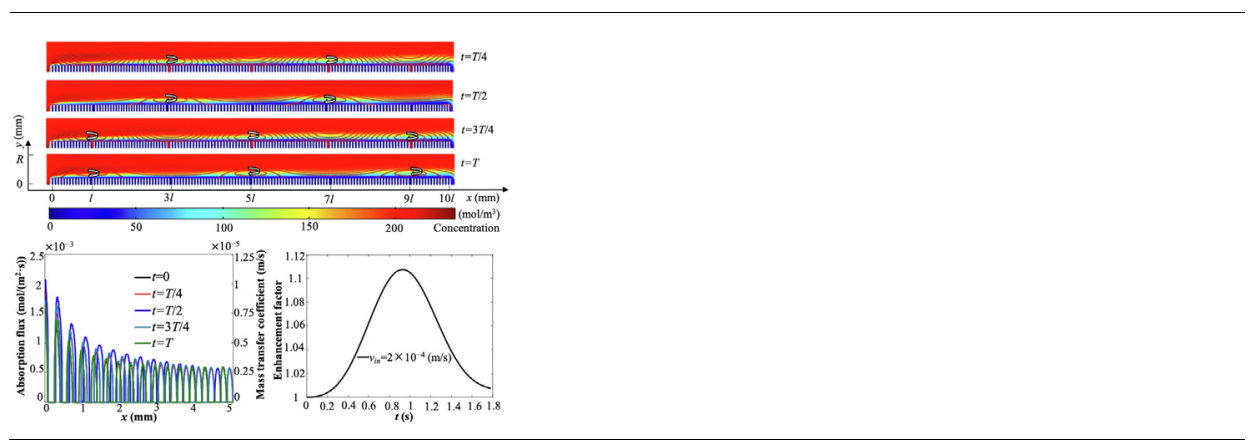

\section{A B S T R A C T}

Given the complex motility of the intestinal wall featuring villous structure, the mechanisms by which the motility can enhance mass transfer and absorption in the small intestine have been a mystery. In this work, the challenging task to couple the intestinal lumen flow and the contraction movement of the villi is tackled by a multi-physics model. The effects of motility of an entire duodenum on the mass transfer and absorption under the influence of ambient flow can be systematically explored. It is revealed that the top of the villi plays a major role in mass absorption. For a rat duodenum, the pendular movement can enhance mass transfer and absorption by up to about $72 \%$. With the introduction of ambient flow, although the absorption amount increases, the enhancement of mass transfer by pendular activity is inhibited. Specifically, the average enhancement factor decreases sharply from $\sim 1.35$ to $\sim 1.05$.

(c) 2019 Elsevier Ltd. All rights reserved.

\section{Introduction}

Obesity and diet-related chronic diseases including type-2 diabetes, cardiovascular disease, hypertension, hyperglycemia and hyperlipidemia have become a significant and growing public health issue worldwide (Fonseca, 2012; Moxon et al., 2016). To

\footnotetext{
* Corresponding author.

E-mail address: jie.xiao@suda.edu.cn (J. Xiao).
}

effectively address these issues, digestion and absorption process in the human body must be taken into account for rational formulation design of functional foods and drugs, so that nutrients or functional molecules can be delivered at desired time and location (Bakalis et al., 2009; Gouseti et al., 2014; Bornhorst et al., 2016; Lentle, 2018).

It is widely recognized that the small intestine is a most important organ for digestion and absorption. The proximal part of the small intestine, i.e., the duodenum which receives pancreatic juice and bile and mixes with partially digested food from the stomach, 


\section{Nomenclature}

\begin{tabular}{|c|c|}
\hline Symbols & \\
\hline$C_{i}$ & concentration of species $i\left(\mathrm{~kg} \mathrm{~m}^{-3}\right)$ \\
\hline$C_{i n}$ & inlet concentration $\left(\mathrm{kg} \mathrm{m}^{-3}\right)$ \\
\hline$D_{i}$ & diffusion coefficient of species $i\left(\mathrm{~m}^{2} / \mathrm{s}\right)$ \\
\hline $\boldsymbol{F}$ & volume force vector $\left(\mathrm{N} / \mathrm{m}^{3}\right)$ \\
\hline $\boldsymbol{I}$ & unit vector $(-)$ \\
\hline $\mathbf{N}_{i}$ & mass flux of species $i\left(\mathrm{~kg} \mathrm{~m}^{-2} \mathrm{~s}^{-1}\right)$ \\
\hline$p$ & pressure $(\mathrm{Pa})$ \\
\hline $\boldsymbol{u}$ & fluid velocity $\left(\mathrm{m} \mathrm{s}^{-1}\right)$ \\
\hline$v$ & velocity of intestinal wall motility $\left(\mathrm{m} \mathrm{s}^{-1}\right)$ \\
\hline$N$ & total number of villi over an area of $2 l \times 2 l$ \\
\hline$n$ & number of villi over a length of $2 l$ \\
\hline $2 l$ & length of a section (mm) \\
\hline$L$ & length of the duodenum (mm) \\
\hline$R$ & radius of the duodenum (mm) \\
\hline$h$ & length of a villus (mm) \\
\hline$d$ & width of a villus (mm) \\
\hline$w$ & distance between two adjacent villi (mm) \\
\hline$f$ & frequency of the pendular activity (Hz) \\
\hline$A_{m}$ & maximum amplitude of the strain $\left(\mathrm{s}^{-1}\right)$ \\
\hline$\underline{K}$ & mass transfer coefficient $\left(\mathrm{m} \mathrm{s}^{-1}\right)$ \\
\hline K & average mass transfer coefficient $\left(\mathrm{m} \mathrm{s}^{-1}\right)$ \\
\hline
\end{tabular}

$\bar{K}^{*} \quad$ average mass transfer coefficient for the case without movement $\left(\mathrm{m} \mathrm{s}^{-1}\right)$

$\mathscr{L} \quad$ total length of the rough surface in 2D (mm)

$m$ amount of nutrients absorbed $\left(\mathrm{mol} \mathrm{m}^{-1}\right)$

$v_{\text {in }} \quad$ inlet velocity $\left(\mathrm{m} \mathrm{s}^{-1}\right)$

\section{Greek Letters}

$\omega \quad$ density of villi(number $/ \mathrm{mm}^{2}$ )

$\mu \quad$ fluid viscosity (Pa s)

$\rho \quad$ density $\left(\mathrm{kg} \mathrm{m}^{-3}\right)$

$\dot{\varepsilon} \quad$ longitudinal strain rate $\left(\mathrm{s}^{-1}\right)$

$\alpha \quad$ enhancement factor

$\bar{\alpha} \quad$ average enhancement factor

$\delta \quad$ length of the rough surface in 2D (mm)

$\begin{array}{ll}\text { Subscript } & \\ a & \text { total } \\ b & \text { bulk } \\ m & \text { max } \\ s & \text { surface }\end{array}$

plays a vital role in food processing, both physically and chemically (Riahi and Roy, 2011; Schulze, 2015; Wright et al., 2016). Some researchers further revealed that nutrients need to be transported to the intestinal wall before being absorbed through the epithelial cells of the intestinal villi. Hence, the villi play a critical role in the process of digestion and absorption (Wang et al., 2010; Lim et al., 2014).

Over the past decades, significant efforts have been devoted to the experimental and simulation study of the digestion and absorption process in the small intestine. As far as experimental research is concerned, in view of the high cost and ethical constraints associated with in vivo trials, in vitro bionic experiments using gastrointestinal animal and human models are thus an alternative approach (Tharakan et al., 2010; Lentle and Loubens, 2015). As a matter of fact, it is a challenging task to design well-controlled experiments for the digestion and absorption study due to the inherent complexity of the small intestine. Lentle et al. used longitudinal spatiotemporal mapping (Canto et al., 1996; Lentle et al., 2007; Janssen and Lentle, 2013; Lentle et al., 2013) to examine the pendular movement of mucosa and associated villi in the proximal duodenum of the rat. Geometric details and movement pattern of the duodenum and villi were revealed. Lentle and his groups (Lentle and Janssen, 2008; Lim et al., 2013) took the distal ileum of the squirrel as the research object. With the help of a camera and a microscope, they recorded the movements of the mucosa and associated villi. Moreover, the contraction of longitudinal muscles was characterized using strain rate mapping. The length and width of the villi as well as their density, and hence their movement pattern were also reported. This provides a certain reference value for scholars engaged in simulating the contraction movement of the small intestine, such as the geometric distribution of the intestinal wall villi and the verification of the villi contraction movement. However, visualization of the flow pattern in the intestine could hardly be realized in experiments. As a result, the influence of villi motion on the flow field and hence mass transfer in the intestinal lumen remains a mystery. In this regard, transport theory together with numerical simulation is a powerful tool to reveal these mysterious phenomena.

Many computational efforts can be identified as well. Stoll et al. (2000), Jeffrey et al. (2003) used state-of-the art numerical meth- ods to explore the fluid flow in the lumen driven by the peristaltic contractions of the wall. Their work provides new insights into the pressure and flow profiles in the lumen and quantifies shear stresses between lumen and wall. However, whether the enhancement of mass transfer can be assessed during non-propagating longitudinal contraction (i.e., pendular activity) of the intestinal wall is yet to be known. De Loubens and his groups (Lentle et al., 2013) approximated the gut lumen as a smooth cylinder and found that 'real-time' non-propagating longitudinal contractions could offer in-situ localized and weak mixing of viscous fluids and augment mass transfer in the vicinity of gut wall. Their seminal work considered a whole rat duodenum and investigated how wall movement could lead to nutrient mixing and mass transfer in the intestinal lumen. However, in their numerical model, the intestinal wall was assumed to be a smooth surface without villi. Moreover, nutrient absorption was not explored.

Listed above are the modeling work focusing on the whole duodenum at macroscale. Some other papers discussed the influence of the villi movement on the local flow field and the mass transfer from a microscopic point of view. Loubens and his groups (Lim et al., 2015) used the lattice-Boltzmann model (LBM) to gain an in-depth understanding of the effects of villi movement on the flow, as well as mixing and absorption of nutrients in the intestinal lumen. However, the investigated system was just a very small section of the duodenal lumen involving few villi only. The bulk flow in the lumen induced by the global motility of the intestinal wall was not taken into account. Localized phenomena captured by their model may not be sufficient to understand digestion and absorption in the whole duodenum.

Brasseur and his group (Wang et al., 2010) used a twodimensional (2D) LBM method to simulate villi movement and fluid flow. The unique contribution of their work is to couple "micro" flow caused by coordinated motions of mucosal villi with lumen-scale "macro" flow generated by gut motility. It was found that microscale contraction motions of villi can enhance the macroscopic advective flux and hence the nutrient absorption rate by creating a 'micro-mixing layer'. In 2017, they further extended the model to 3D (Wang and Brasseur, 2017). Their findings demonstrated the necessity of coupling microscale flow (induced by villi motion) and macroscale bulk flow (driven by gut motility). They 
also pointed out the potential importance of villi movement in the intestinal absorption. However, the adoption of the lid-driven cavity flow to approximate the macroscale bulk flow remains questionable. Moreover, the villi scale models developed so far assumed simplified villi movement (Lim et al., 2015; Wang et al. 2010), which lacks sound connection with motility.

Most studies of mixing driven by intestinal motility simplified intestinal lumen surface as a smooth wall without villi. Many of them investigated non-propagating circular contractions, i.e. segmentation contractions (Grivel and Ruckebusch, 1972; Gwynne et al., 2004), and propagating coordinated circular and longitudinal contractions, i.e. peristaltic contractions (Schulze-Delrieu, 1999) that propel the luminal content along the small intestine (Janssen et al., 2007). Mixing generated by non-propagating longitudinal contractions, i.e. pendular contractions (Christensen, 1971; Lammers et al., 1996; Melville et al., 1975) has not been rigorously investigated.

In this work, a multi-physics model is developed to simulate mass transfer and absorption in a duodenum. The unique features of the model are three-folds: (1) the simulation of a complete rat duodenum with its hierarchical structure taken into account allows a holistic understanding of mass transfer and absorption mechanisms, (2) the motility of the duodenum together with its villi is implemented using the moving mesh method, (3) the influence of villi movement on mass transfer and absorption can be evaluated under different bulk flow conditions. Analysis methods are also developed to quantify the enhancement of mass transfer and absorption by gut motility, specifically the pendular movement investigated in this work.

\section{Modeling and analysis methods}

\subsection{Geometry construction and simplification}

The duodenum is approximated as a tube with a radius of $R$ ( $\mathrm{mm}$ ) and a length of $L(\mathrm{~mm})$ between the pylorus and the pancreatic duct (see Fig. 1(a)). Finger shaped villi are uniformly distributed on the inner surface of the tube. As a first step towards 3D simulation of the system in Fig. 1(a) with tremendous amounts of villi, this work investigates the 2D system in Fig. 1(b), which is the cross-section of the tube and associated villi. A 2D villus is a rectangle of width $d$ with a round cap (Fig. 1(b)). It is assumed that villi are arrayed uniformly over the inner surface of the wall. To implement pendular movement of the wall, the proximal duodenum should be divided into 10 sections with each section of length $l$ (de Loubens et al., 2013). The total number of villi over an area of $2 l \times 2 l$ can be calculated as:

$N=4 l^{2} \omega$

where $\omega$ is the area density of villi (i.e. number of villi per $\mathrm{mm}^{2}$ area of gut mucosa). For the cross-sectional 2D view in Fig. 1(b), the number of villi over a length of $2 l$ is:

$n=\sqrt{N}=2 l \sqrt{\omega}$

The total widths of $n$ villi is given by:

$d_{a}=n d$

where $d$ is the width of a single villus. The intervillous space between two adjacent villi becomes:

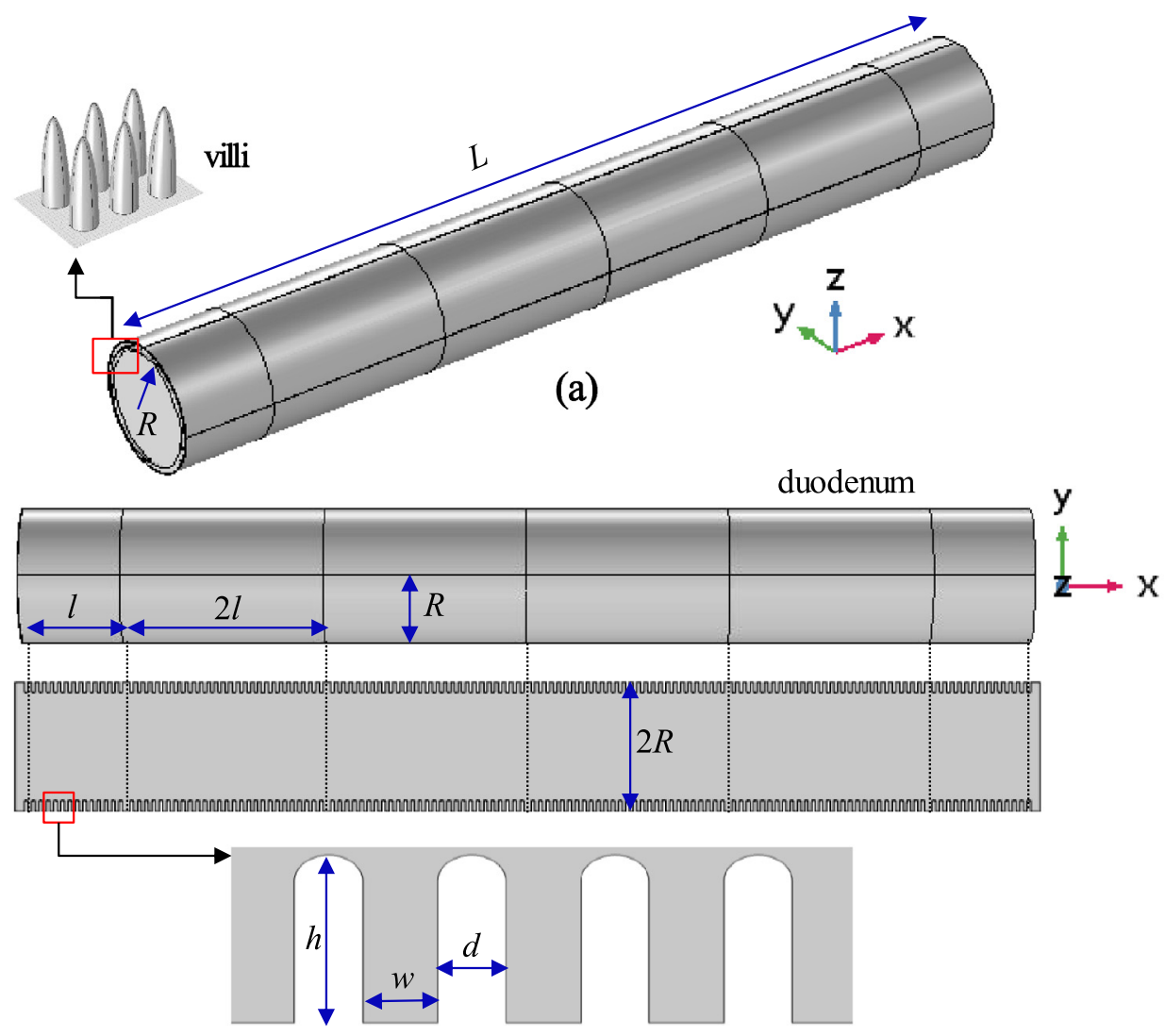

(b)

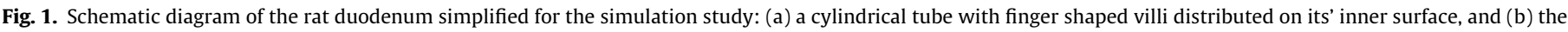
cross-sectional view of the tube with associated villi, which is the simplified 2D system simulated in this work. 
$w=\frac{2 l-n d}{n+1}=\frac{2 l-2 l d \sqrt{\omega}}{2 l \sqrt{\omega}+1}$

\subsection{Governing equations}

In this study, transport of nutrients is coupled with fluid flow, and nutrient absorption is implemented as a boundary condition. The flow in the duodenum is a laminar flow described by the continuity equation and the Navier-Stokes equation for an incompressible fluid.

$\rho \nabla(u)=0$

$\rho \frac{\partial u}{\partial t}+\rho(u \cdot \nabla) u=\nabla \cdot\left[-p I+\mu\left(\nabla u+(\nabla u)^{T}\right)\right]+F$

where $\rho$ is the density of the fluid $\left(\mathrm{kg} / \mathrm{m}^{3}\right)$; $u$ is the fluid velocity $(\mathrm{m} /$ $\mathrm{s}) ; p$ is pressure (Pa); $I$ is a unit vector; $\mu$ denotes the viscosity of the fluid (Pa.s); and $F$ is the volume force vector $\left(\mathrm{N} / \mathrm{m}^{3}\right)$.

Advective and diffusive transport of nutrients is described by:

$\frac{\partial c_{i}}{\partial t}+\nabla \cdot\left(-D_{i} \nabla c_{i}\right)+u \cdot \nabla c_{i}=0$

$N_{i}=-D_{i} \nabla c_{i}+u c_{i}$

where $D_{i}$ is the diffusion coefficient for species $i\left(\mathrm{~m}^{2} / \mathrm{s}\right) ; c_{i}$ is the concentration of species $i\left(\mathrm{~kg} / \mathrm{m}^{3}\right) ; u$ is the flow velocity $(\mathrm{m} / \mathrm{s})$ obtained by solving Eqs. (5) and (6); $N_{i}$ is the mass flux $\left(\mathrm{kg} /\left(\mathrm{m}^{2} \cdot \mathrm{s}\right)\right)$. Glucose, as a key carbohydrate for most living organisms, is investigated in this work. The diffusion coefficient of glucose at 25 is $6.7 \times 10^{-10}\left(\mathrm{~m}^{2} / \mathrm{s}\right)$ (Gouseti et al., 2014). Based on the Stokes-Einstein equation, at the body temperature, the diffusion coefficient of glucose in water becomes $8.97 \times 10^{-10}\left(\mathrm{~m}^{2} / \mathrm{s}\right)$.

\subsection{Boundary and initial conditions}

As described in the last section, coupled momentum and mass transfer phenomena have been modeled. The boundary conditions for these two physics are indicated in Fig. 2.

It is assumed that constant nutrient supply comes from stomach through pylorus. The fluid with a constant glucose inlet concentration, $C_{i n}$, flows into the intestine at a fixed inlet velocity of $v_{i n}$. The outlet gauge pressure is set to $0 \mathrm{~Pa}$. Non-slip boundary conditions are applied to the wall of the duodenum (including the surface of villi). It is assumed that the nutrient transfer and absorption process in the duodenum is a diffusion controlled process. Thus, the surface concentration of the glucose at the duodenum inner wall is set to zero, implying that glucose can be instantaneously absorbed once reaching the inner surface of the duodenum. The initial glucose concentration and fluid velocity in the lumen are both zero. For a 2D system, only half of the lumen should be investigated due to the symmetrical geometry.

The challenging task in this work is to simulate mass transfer and absorption in a continuously changing geometry. The movement of duodenum wall together with associated villi is implemented using the moving mesh method. A velocity, $v$, is specified for the duodenum inner surface (including the villi surface). The mathematical expression of velocity can be determined by the motility of the duodenum, which is derived in the next section.

\subsection{Motility specification: simplified pendular activity}

The motility of duodenum leads to the change of the simulation domain. The simplified pendular activity caused by the longitudinal contraction of the muscle is investigated in this work. As shown in Fig. 3, the cyclic evolution of the longitudinal strain rate $\dot{\varepsilon}$ along the axial direction can be expressed by a sinusoidal function:

$\dot{\varepsilon}(x, t)=A(x) \sin (2 \pi f t)=A(x) \sin \left(2 \pi \frac{t}{T}\right)$

where $\dot{\varepsilon}$ is the rate of strain with a positive value (i.e. $\dot{\varepsilon}>0$ ) indicating local lengthening and a negative value (i.e., $\dot{\varepsilon}<0$ ) indicating local shortening; $f$ is the cyclic frequency of the pendular activity, whose period is $T$; $t$ is time. $A(x)$ is the maximum amplitude of the strain rate (de Loubens et al., 2013), which changes along the axial direction (see Fig. 2(b)):

$A(x)=A_{m} \cos \left(2 \pi \frac{x}{4 l}\right)=A_{m} \cos \left(\pi \frac{x}{2 l}\right)$

Fig. 3 shows that the shortening section and the lengthening section, each of length $2 l$, are adjacent to each other. Within one cycle, the same section experiences both shortening and lengthening (see Fig. 3(b)). The velocity of the movement of the wall with associated villi can be obtained by integrating the longitudinal strain rate along the axial length.

$v(x, t)=\int_{0}^{x} \dot{\varepsilon}(x, t) d x$

Plugging Eqs. (9) and (10) into Eq. (11) yields:

$v(x, t)=\int_{0}^{x} A_{m} \sin \left(2 \pi \frac{t}{T}\right) \cos \left(\pi \frac{x}{2 l}\right) d x$

After integration, the velocity becomes:

$v(x, t)=\frac{2 l}{\pi} A_{m} \sin \left(\frac{\pi}{2 l} x\right) \sin (2 \pi f t)$

And the maximum velocity of intestinal wall movement is:

$v_{m}=\frac{2 l}{\pi} A_{m}$

The velocity in Eq. (13) is then specified for the moving wall to implement the pendular activity.

\subsection{Quantification and analysis of mass transfer and absorption}

The mass transfer coefficient $(K)$ can be calculated to quantify the significance of nutrient transfer from bulk to the absorption surface in the lumen. It is given by:

$K=J /\left(C_{b}-C_{s}\right)$

where $J$ is the flux of the nutrient absorbed through the intestinal wall $\left(\mathrm{mol} \cdot \mathrm{m}^{-2} \cdot \mathrm{s}^{-1}\right) ; C_{b}\left(\mathrm{~mol} / \mathrm{m}^{3}\right)$ is the bulk concentration, which is equal to the inlet concentration; $C_{S}\left(\mathrm{~mol} / \mathrm{m}^{3}\right)$ is the concentration

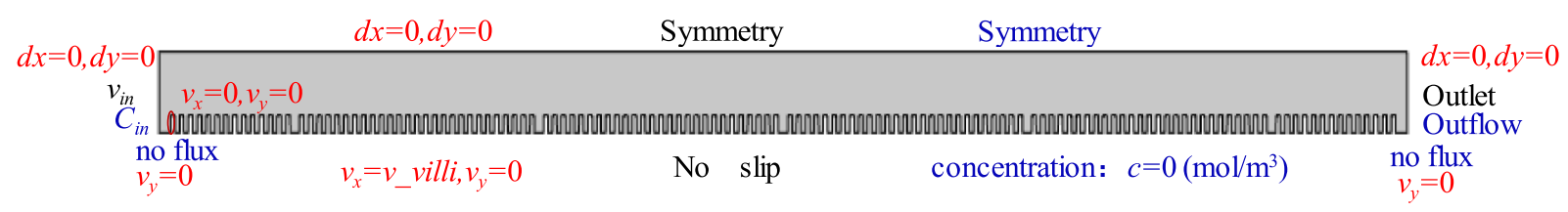

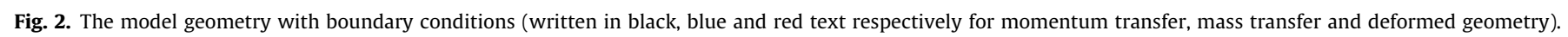




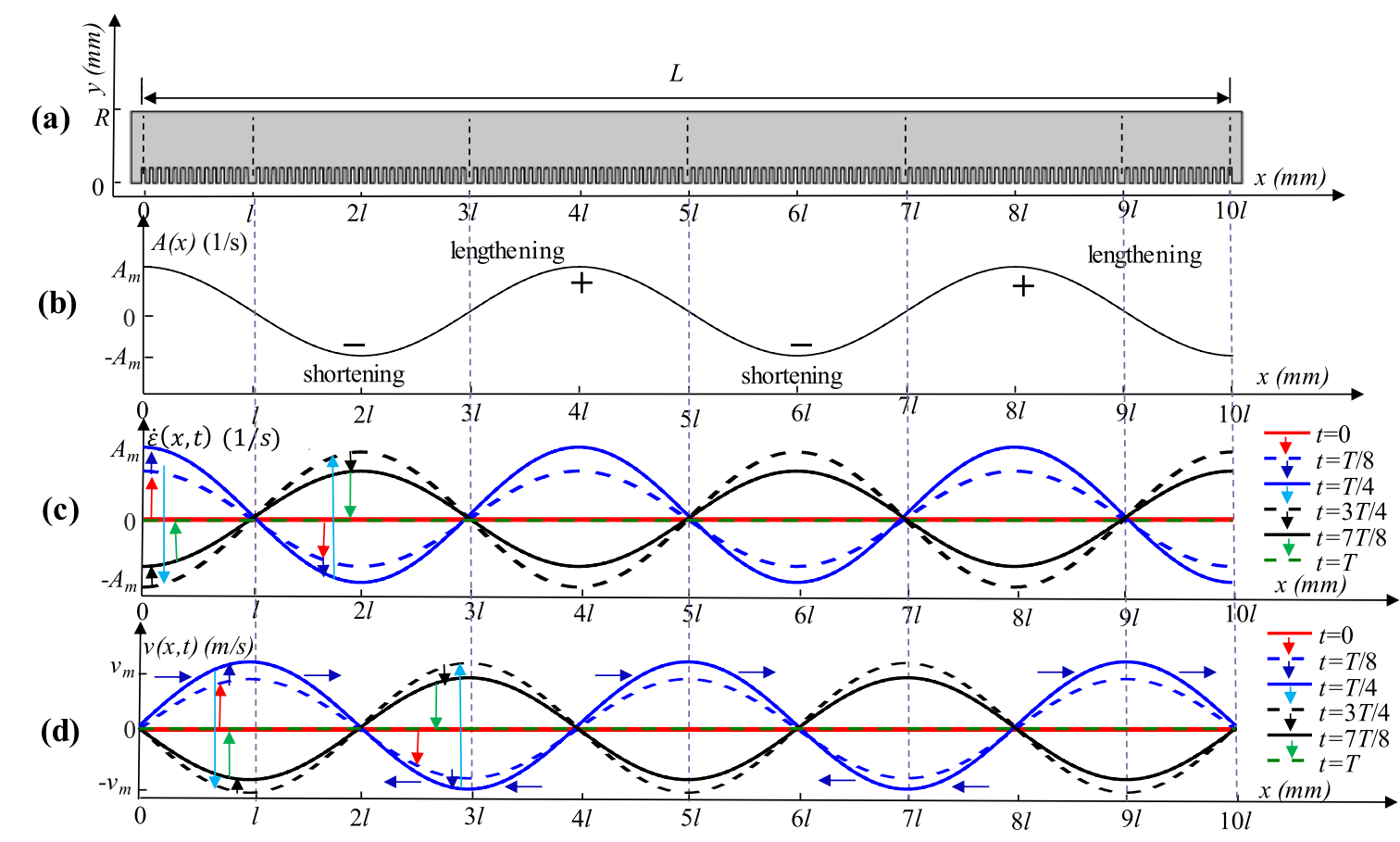

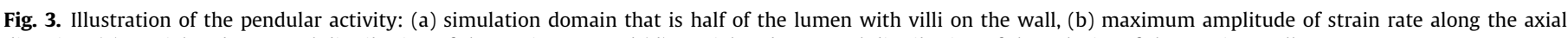
direction, (c) spatial and temporal distribution of the strain rate, and (d) spatial and temporal distribution of the velocity of the moving wall.

of the nutrient on the absorption surface, i.e., the inner wall of the duodenum. For a diffusion controlled process, $C_{s}$ is zero.

It is understandable that the nutrient flux is not uniformly distributed on the rough absorption surface (see Fig. 2), which holds for the mass transfer coefficient as well. An average mass transfer coefficient can be derived as:

$\bar{K}(t)=\frac{\int_{0}^{\mathscr{L}} K d \delta}{\int_{0}^{\mathscr{L}} d \delta}$

where $\mathscr{L}$ is the total length of the rough surface in $2 \mathrm{D}$.

The enhancement of mass transfer due to the pendular activity can be quantified by an enhancement factor, which is defined as:

$\alpha(t)=\frac{\bar{K}(t)}{\bar{K}^{*}}$

where $\overline{K^{*}}$ is the average mass transfer coefficient for the case without pendular movement of the wall.

Averaging the enhancement factor over time in one cycle offers an average enhancement factor:

$\bar{\alpha}=\frac{\int_{0}^{T} \alpha d t}{T}$

The amount of nutrients absorbed within time $t$ can be obtained through integration of the flux over the absorption surface and over time.

$m(t)=\int_{0}^{t} \int_{0}^{\mathscr{L}} J d \delta d t$

For a $2 \mathrm{D}$ system, $m$ is the amount of nutrients absorbed per unit length with a unit of $\mathrm{mol} / \mathrm{m}$.

\subsection{Simulation procedure}

The focus of this work is to quantitatively evaluate the effect of pendular activity on mass transfer and absorption in the intestinal lumen. A two-step simulation scheme is designed. The first step is a steady state simulation of mass and momentum transport in the lumen with a fixed geometry, i.e., without the pendular activity. After that, the moving mesh model is activated to implement the pendular movement of the intestinal wall. A transient solver is used to solve coupled mass and momentum transfer in a timevariant geometry. The solution from the 1 st step steady-state solver becomes the initial condition for the 2 nd step transient solver. All the governing equations are solved using Comsol Multiphysics software (COMSOL, xxxxCOMSOL, 2018).

\section{Results and discussion}

The duodenum of the rat is selected in this work to demonstrate the efficacy of the introduced method. Detailed geometry of the lumen and villi are listed in Table 1 . The parameter values for the pendular activity are also given.

\subsection{Mesh independence study}

Six different meshes have been tested for the base case study, whose settings are given in Table 1 . The number of grids ranges from 7795 to 85785 . As shown in Fig. 4, different from the coarse grids in bulk, fine grids are used to capture the sharp gradients adjacent to the absorption surface.

As can be seen from Fig. 5, increasing the number of grids from 7795 to 33,987 leads to significant differences in the enhancement factor profile and the mass absorption profile. Further increasing the number of grids to 71,317 results in slight change of the enhancement factor. Negligible differences can be identified between the scheme with 71,317 grids and the scheme with 85,785 grids. The computational time increases with the number of grids in the mesh. All simulations were carried out on one node of a high performance computing cluster, which has the Intel (R) Xeon (R) CPU (E5-2660 v4 @ 2.00 GHz and 14 cores). Running cases in Fig. 4 costed respectively 191 s, 239 s, 761 s, 1515 s, 1803 s, and 
Table 1

Parameter values of the pendular activity for a rat duodenum.

\begin{tabular}{|c|c|c|c|}
\hline Parameters & Notation & Value & References \\
\hline Length of a section & $2 l$ & $9 \mathrm{~mm}$ & (Lentle et al., 2012) \\
\hline Length of the duodenum & $L$ & $45 \mathrm{~mm}$ & (Lentle et al., 2012) \\
\hline Radius of the duodenum & $R$ & $3 \mathrm{~mm}$ & (Lim, 2015) \\
\hline Length of a villus & $h$ & $0.667 \mathrm{~mm}$ & (Holm et al., 2001) \\
\hline Width of a villus & $d$ & $0.156 \mathrm{~mm}$ & (Guseinov and Guseinova, 2008) \\
\hline Density of villi & $\omega$ & $9 \mathrm{~mm}^{-2}$ & (Barry, 1976) \\
\hline Distance between two adjacent villi & $w$ & $0.171 \mathrm{~mm}$ & Eq. (4) \\
\hline Frequency of the pendular activity & $f$ & $0.56 \mathrm{~Hz}$ & (Lentle et al., 2012) \\
\hline Maximum amplitude of the strain rate & $A_{m}$ & $0.21 \mathrm{~s}^{-1}$ & (Lentle et al., 2012) \\
\hline Maximum velocity of intestinal wall movement & $v_{m}$ & $6.14 \times 10^{-4}(\mathrm{~m} / \mathrm{s})$ & Eq. (14) \\
\hline Inlet concentrations & $C_{i n}$ & $200 \mathrm{~mol} / \mathrm{m}^{3}$ & (Leiper, 2015) \\
\hline Inlet velocity & $v_{\text {in }}$ & $0-2 \times 10^{-4} \mathrm{~m} / \mathrm{s}$ & (Janssen and Lentle, 2013) \\
\hline
\end{tabular}

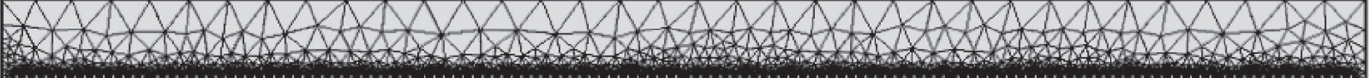

Number of grids:7795

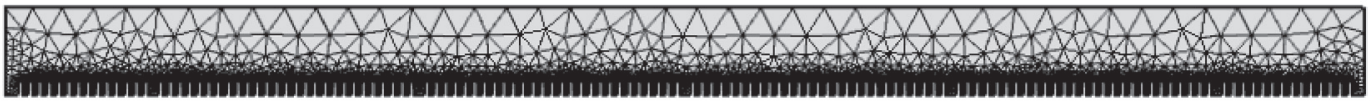

Number of grids:9429

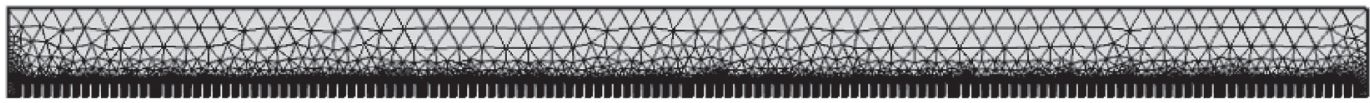

Number of grids:33978

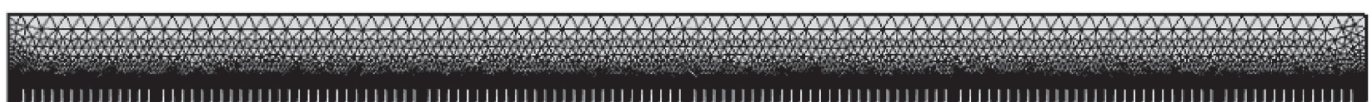

Number of grids:62366

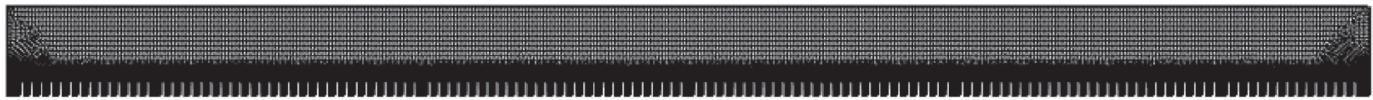

Number of grids:71317

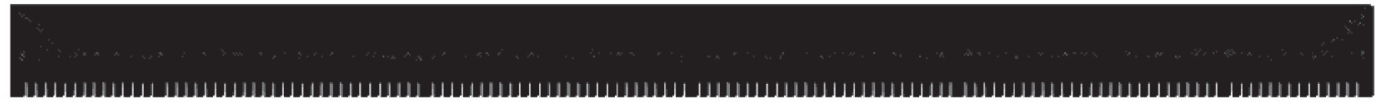

Number of grids:85785

Fig. 4. Six different sets of grids tested in the mesh independence study.

(a)

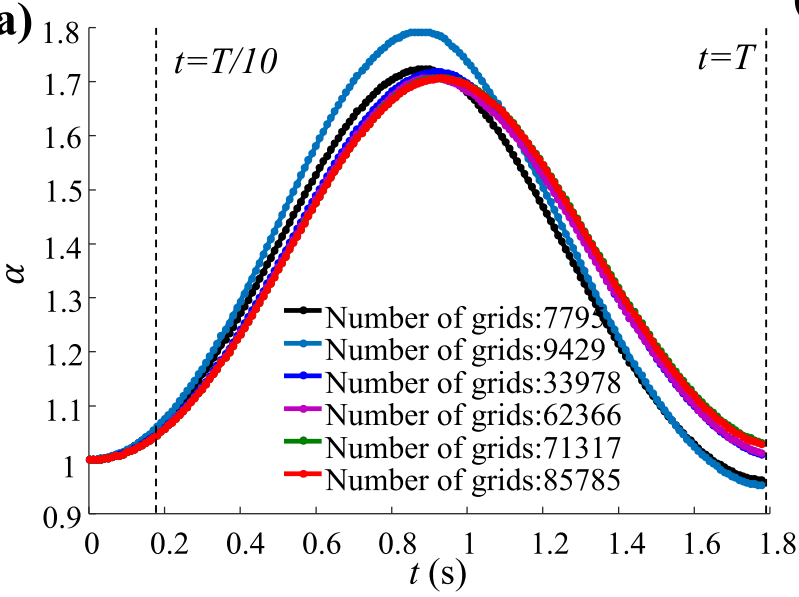

(b)

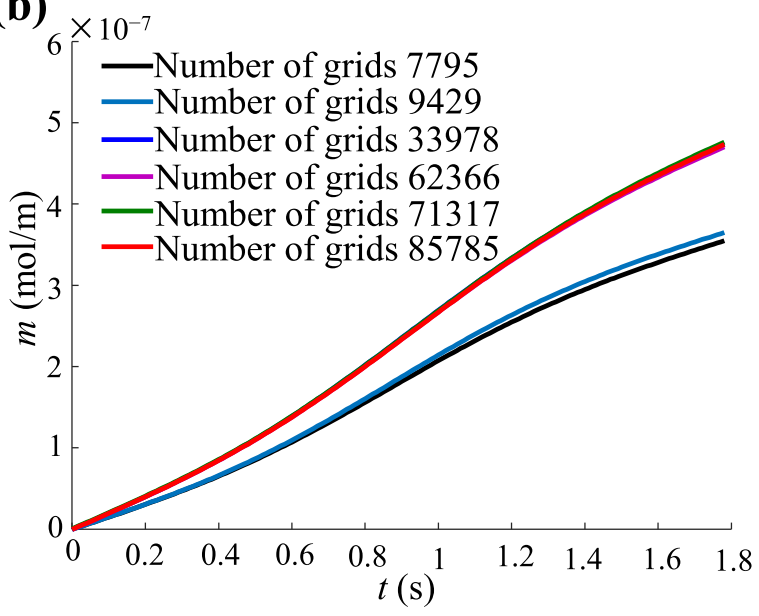

Fig. 5. Comparison of results in one cycle under different meshes: (a) the evolution of enhancement factor, and (b) the evolution of absorbed mass. 
$2455 \mathrm{~s}$. Based on the above analysis, the mesh with 71,317 grids is adopted in this study, as it offers high prediction accuracy with decent computational efficiency.

\subsection{Analysis of flow field and concentration field}

For all cases investigated in this work, one cycle motility process was simulated with a time step of $0.01 \mathrm{~s}$. Multi-physics model provides comprehensive spatial distribution data at any time instant, which can be used to quantify the effect of the motility of the intestinal wall on mass transfer and absorption.

Fig. 6(a) shows the strain rate along the moving wall together with the velocity of the moving wall at four different time instants, which correspond to the four instants in both Fig. 6 (b) and (c). It can be seen that local lengthening and local shortening of the intestinal wall result in flow in both radial and axial directions. Taking the flow field at $t=T / 4$ as an example, within the sections of 0 to $l, 3 l$ to $5 l$, and $7 l$ to $9 l$, the strain rate is positive (see Fig. 6

(a)

( I )

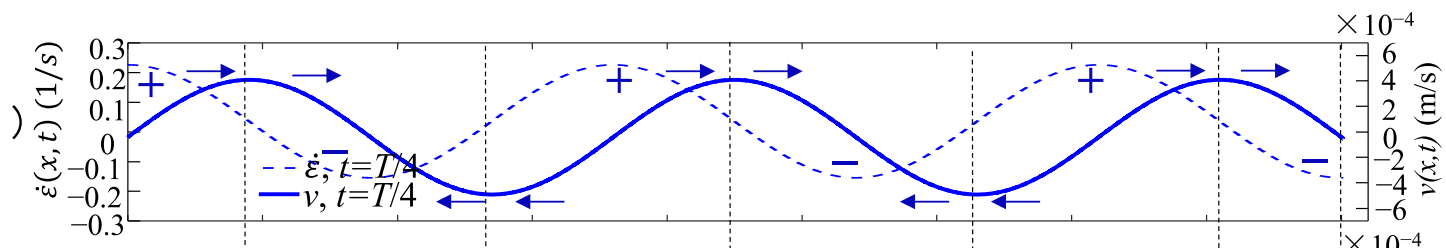

(II)

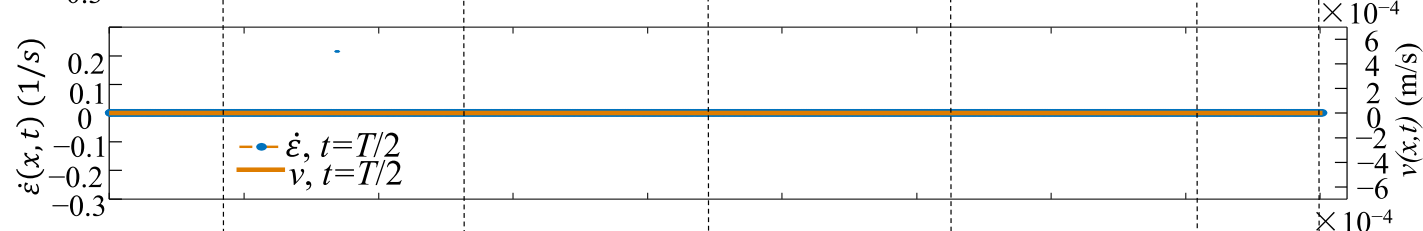

(III)

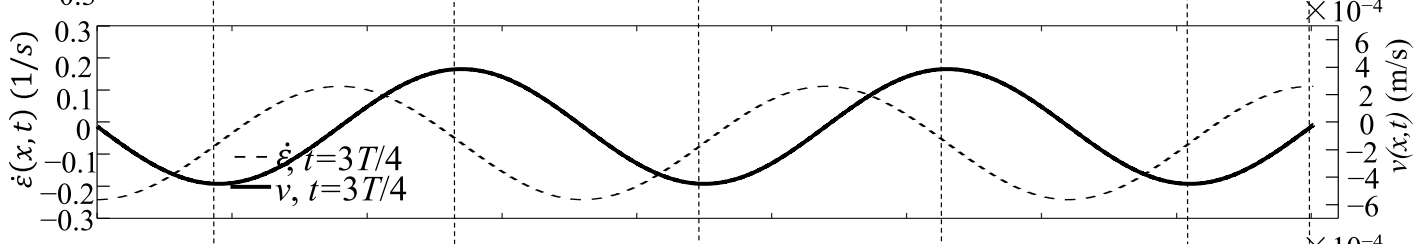

(IV)

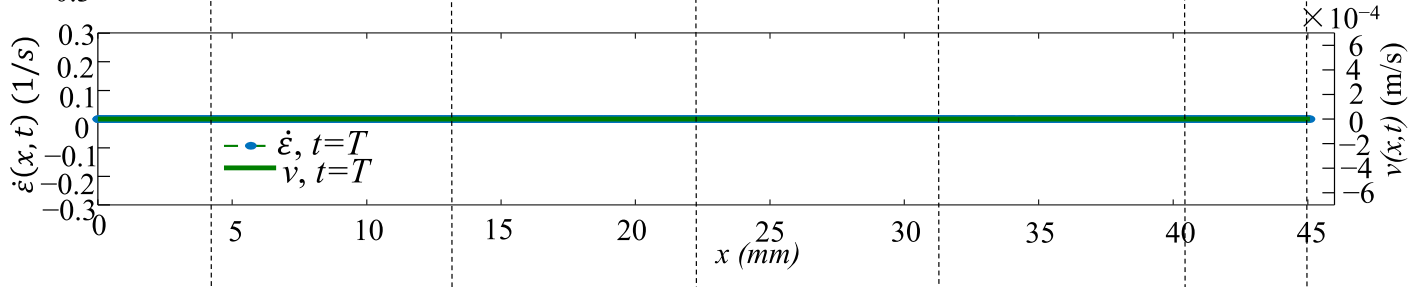

(b)

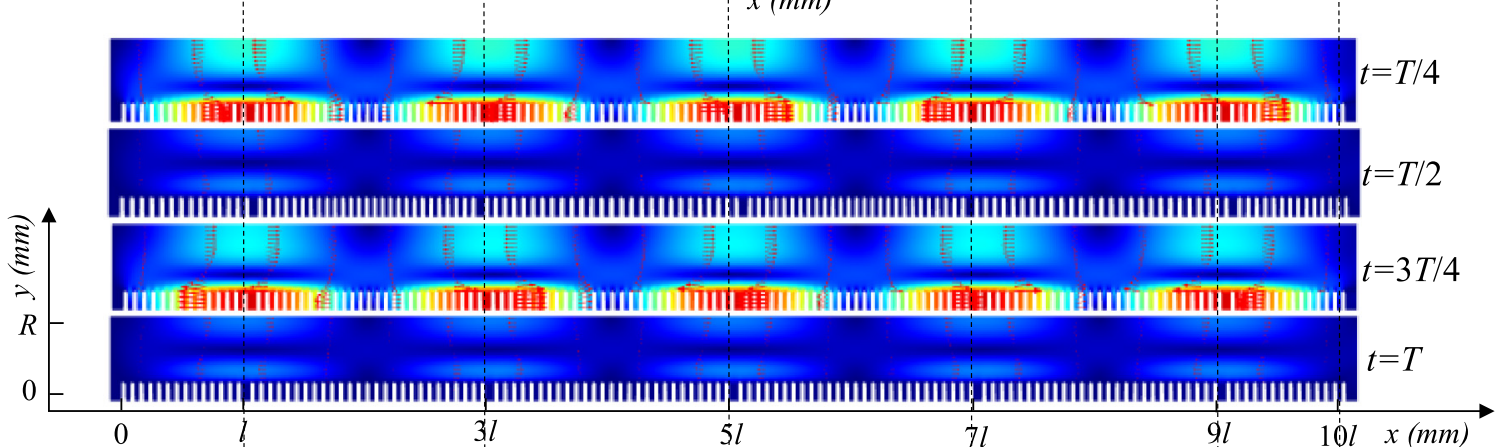

(c)

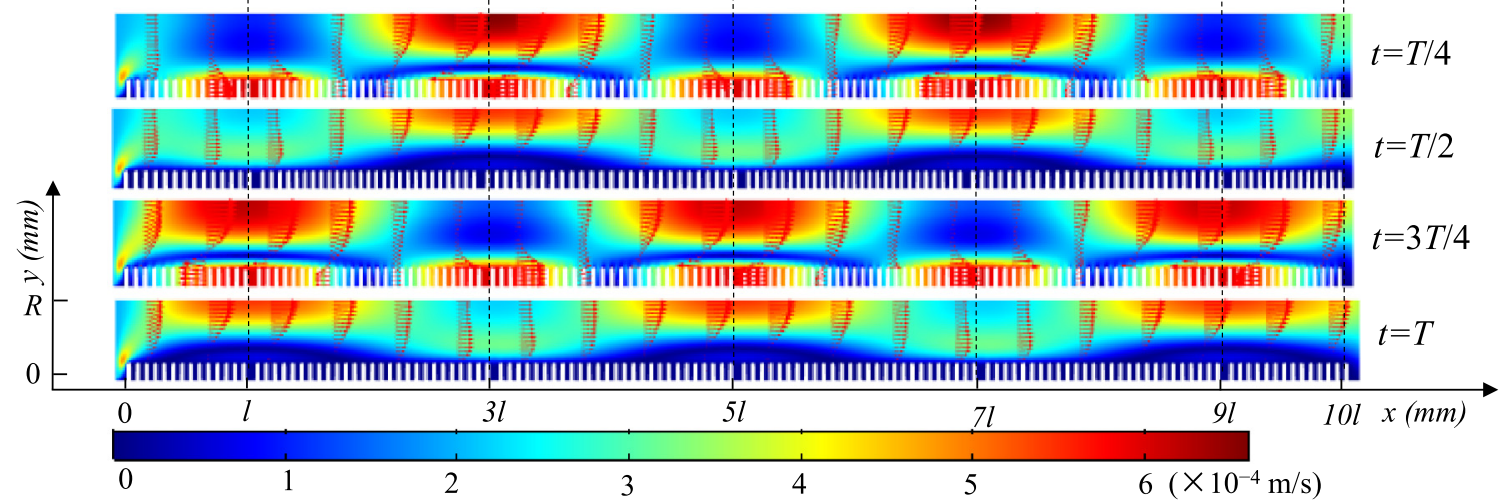

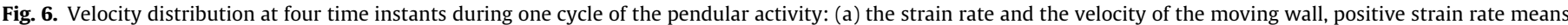

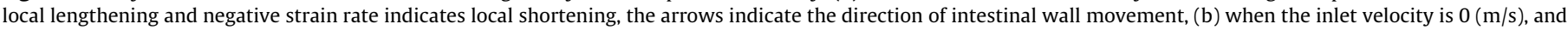
(c) when the inlet velocity is $2 \times 10^{-4}(\mathrm{~m} / \mathrm{s})$. The length of red arrows in (b) and (c) is proportional to the magnitude of velocity. 
(a)). Local lengthening of the wall occurs in these sections, which can be confirmed by the moving direction of the intestinal wall (Fig. 6(a)). The red arrows adjacent to the villi in Fig. 6(b) point into the inter-villous region, implying the nutrient in the intestinal lumen flows into the inter-villous region within the local lengthening sections. On the other hand, local shortening of the wall occurs within the sections of $l$ to $3 l, 5 l$ to $7 l$, and $9 l$ to $10 l$ (see the negative strain rate at $t=T / 4$ in Fig. 6(a)). In Fig. 6(b), the arrows point out from the inter-villous region, indicating the movement of villi pushes fluid out from the inter-villous region. In summary, the pendular activity of the duodenum caused fluid to be drawn into the inter-villous region for the local lengthening section and be driven away from the villi for the local shortening section.

Fig. 6 also shows that the velocity magnitude in the simulated region is relatively large at $t=T / 4$ and $t=3 T / 4$, and relatively small at $t=T / 2$ and $t=T$. This is because the magnitude of wall velocity reaches the maximum value at $T / 4$ and $3 T / 4$, and becomes zero at $T / 2$ and $T$ (see Eq. (13) and Fig. 6(a)). Moreover, local lengthening sections become local shortening ones after $T / 2$, and vice versa. It leads to the directional change of the velocity field after $T / 2$ (see Fig. 6(b)).

After introducing the bulk flow in the axial direction (i.e., the positive $x$ direction), the flow filed becomes quite different (see Fig. 6(b) and (c)). Original velocity in positive $x$ direction is enhanced, while original velocity in negative $x$ direction is inhibited.
The evolution of glucose concentration field during one cycle of the pendular activity is given in Fig. 7, where the streamlines of flow field are also plotted.

It can be seen from Fig. 7(a) that the regular vortex flow is formed by the pendular activity of the intestinal wall. Two adjacent vortexes are opposite in direction, i.e., when a region has a clockwise vortex, it's adjacent region has a counter-clockwise one. There are five vortexes in total and each one covers a length of $2 l$, half of which has local lengthening wall and the other half has local shortening wall. Furthermore, the streamlines are dense when $t=T / 4$ and $t=3 T / 4$, and the streamlines are sparse when $t=T / 2$ and $t=T$, especially in the inter-villous region, which indicates higher velocity existing at $t=T / 4$ and $3 T / 4$. This observation is consistent with the results in Fig. 6(b). The directional change of the velocity field after $T / 2$ leads to the directional change of vortexes (see Fig. 7 (a)). In addition, Fig. 7(a) shows that the concentration gradient mainly exists in the entrance region, that is, near the left boundary of the simulation domain. This phenomenon implies that the axial transport of nutrient cannot be promoted significantly by the vortex flow generated by the pendular activity. The nutrient molecules are restricted in the region of the first vortex and it's quite difficult for them to move across the boundary to the second vortex region.

However, after introduction of the ambient bulk flow, the nutrient almost spreads throughout the entire domain (see Fig. 7(b)). Concentration gradients can be clearly identified above all villi.

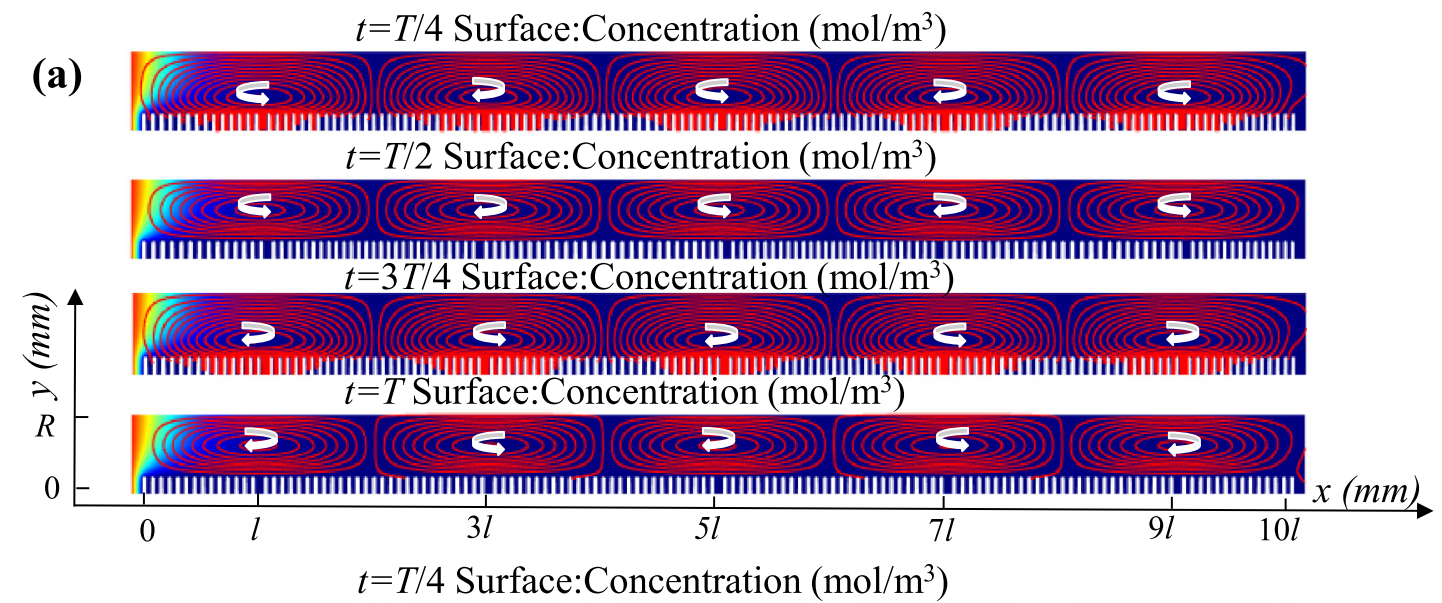

(b)

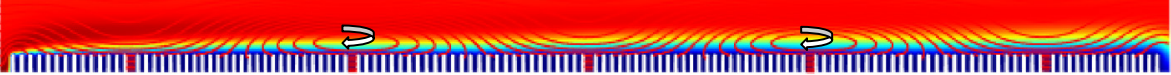
$t=T / 2$ Surface:Concentration $\left(\mathrm{mol} / \mathrm{m}^{3}\right)$

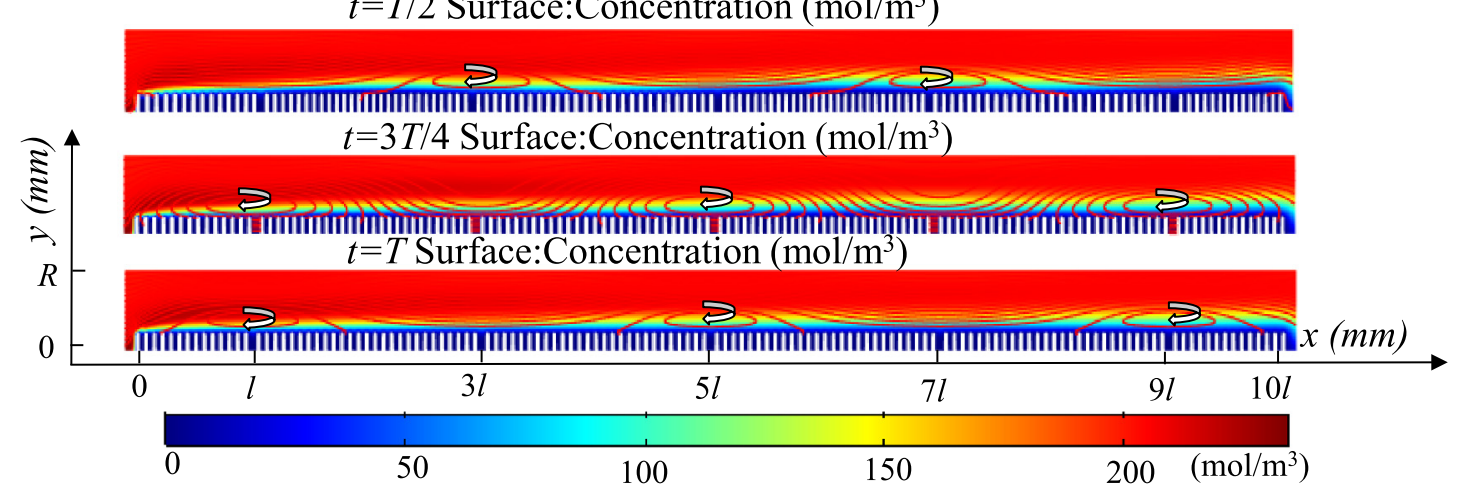

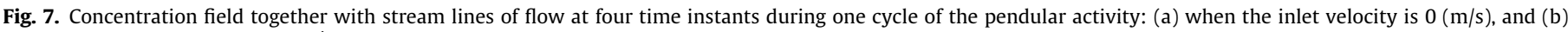
when the inlet velocity is $2 \times 10^{-4}(\mathrm{~m} / \mathrm{s})$. 
At the same time, there are fewer vortexes, specifically in this case, the counterclockwise vortexes have been suppressed.

\subsection{Analysis of mass transfer and absorption}

The distribution of absorbed flux of nutrient on the villi surface during one cycle of the pendular activity can be plotted for further analysis. For a diffusion controlled process in this work, nutrient concentration on the villi surface $\left(C_{s}\right)$ is 0 . According to Eq. (15), the mass transfer coefficient is linearly proportional to the absorption flux, thus they can be plotted in one figure using the same curve but two different axes. A larger mass transfer coefficient indicates a higher absorption flux. Fig. 8(a) shows that, without ambient bulk flow, the absorption mainly occurs in the region between $0 \mathrm{~mm}$ and $9 \mathrm{~mm}$, i.e., $2 l$. The flux becomes negligible beyond $9 \mathrm{~mm}$. It confirms the finding in Fig. 7(a) that nutrient is restricted in the region of the first vortex.

In order to have a clear view of the flux distribution in the entrance region, the section between $0 \mathrm{~mm}$ and $5 \mathrm{~mm}$ is plotted in Fig. 8(b). It's interesting to observe a nonuniform distribution with a number of spikes with peaks and valleys. The number of spikes is equal to the number of villi and the location of the peak of the flux corresponds to the peak of a villus. This phenomenon tells us the protruding structure of villi does affect nutrient absorption. For a villus, although the absorption capability of the valley area is not inferior to that of the peak area (in this case, all nutrient molecules reaching the villi surface, no matter where of the villi, can be absorbed immediately), the top of the villus does has the highest absorption flux indicating its key role in nutrient absorption. The absorption flux in the valley area can be as low as 0 indicating the great difficulty for nutrient molecules to be transported into the inter-villous region. The peak values of flux gradually decrease along $x$ and beyond $4.5 \mathrm{~mm}$ (i.e., $l / 2$ ), they become almost negligible. This finding can be confirmed by the concentration distribution in Fig. 7(a), where concentration gradients mainly exist in the region between 0 to $l / 2$.

In Fig. 8(c), the absorption flux at $T / 4$ is compared with that at $t=0$. Note that the results for the case without pendular activity are the same as the results at $t=0$ in Fig. 8. Local lengthening activity can be identified for this section of wall between 0 to $l / 2$ (see the wider spikes in red at $T / 4$ than the black ones at $t=0$, and the positive strain rate in Fig. 6(a)). The spikes shift to the right,
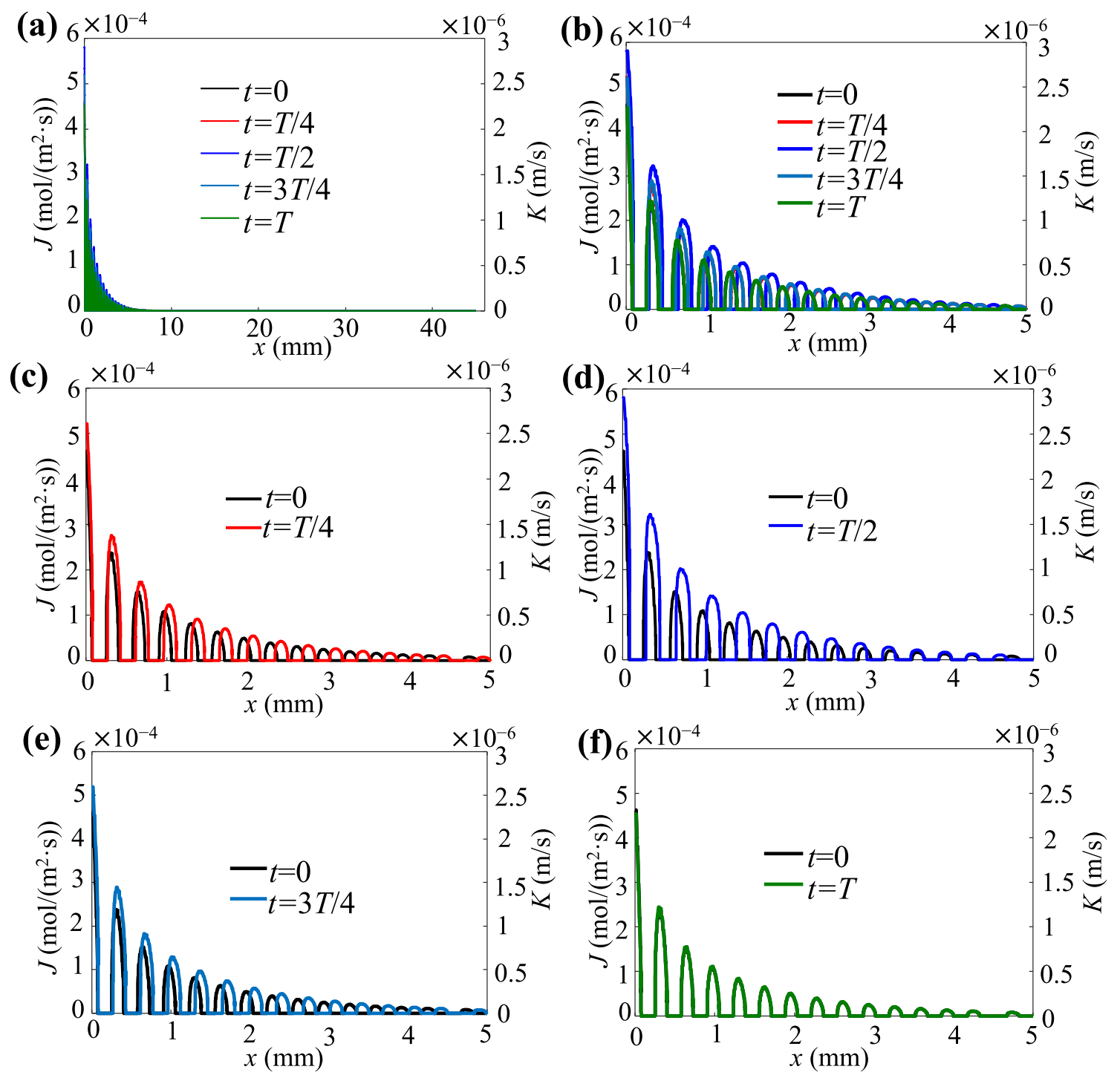

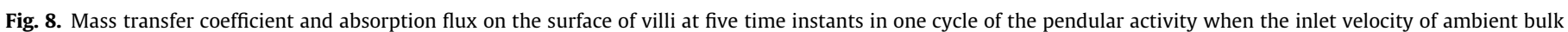

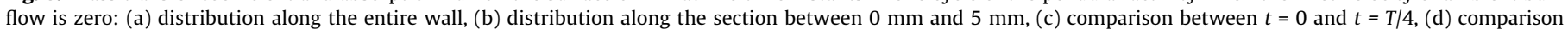
between $t=0$ and $t=T / 2$, (e) comparison between $t=0$ and $t=3 T / 4$, and (f) comparison between $t=0$ and $t=T$. 

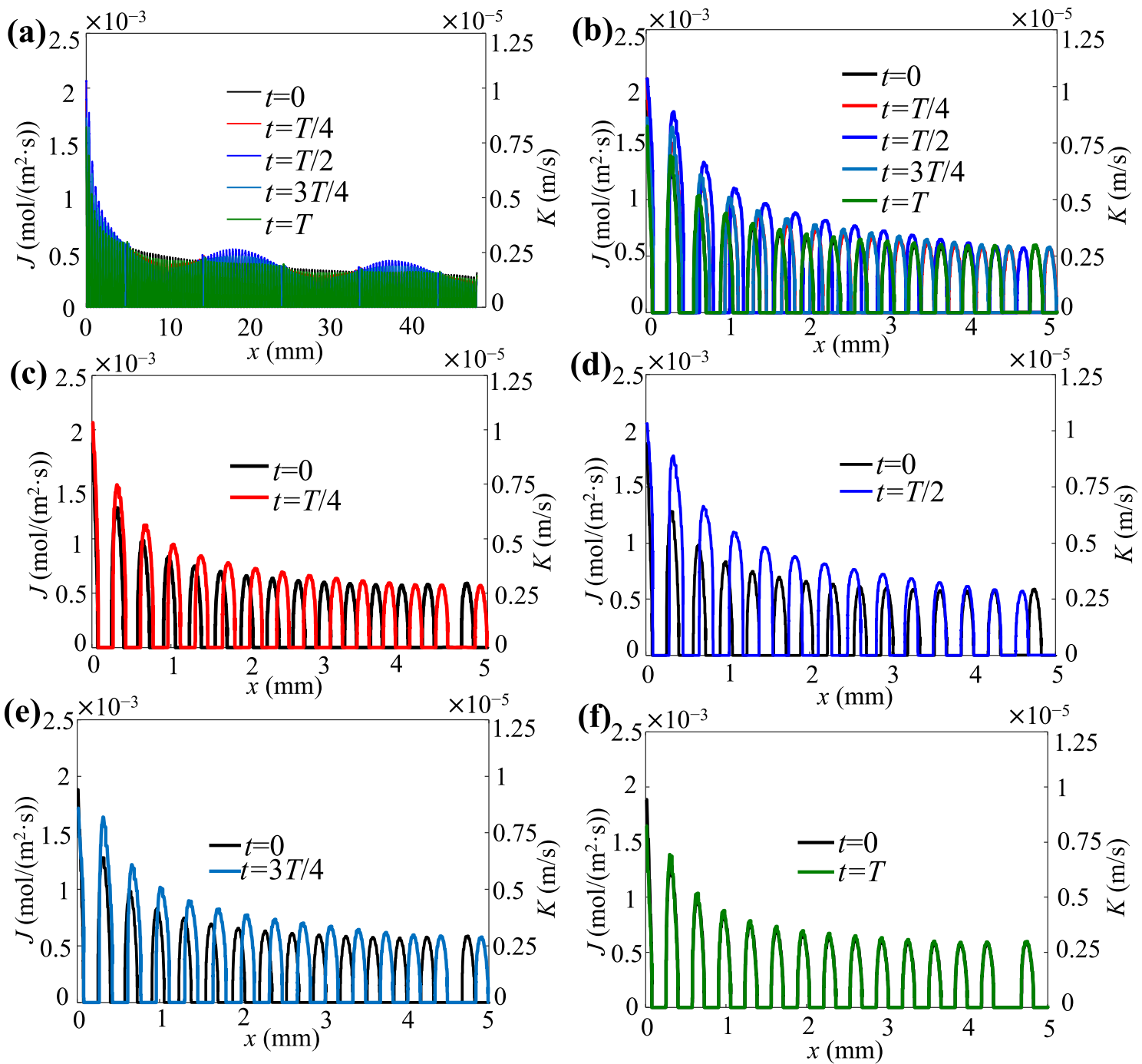

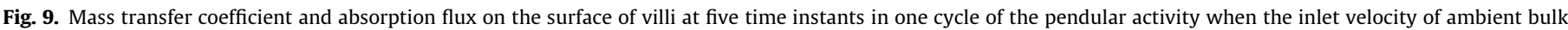

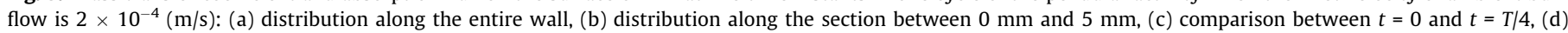
comparison between $t=0$ and $t=T / 2$, (e) comparison between $t=0$ and $t=3 T / 4$, and (f) comparison between $t=0$ and $t=T$.
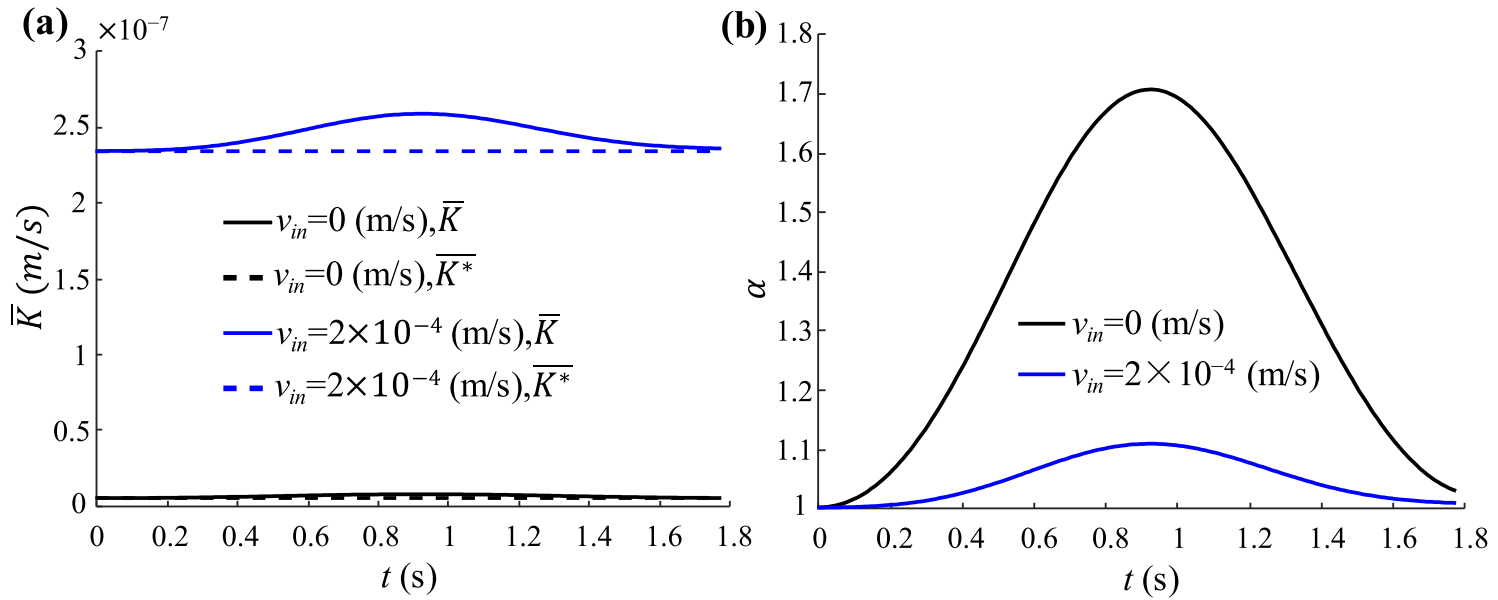

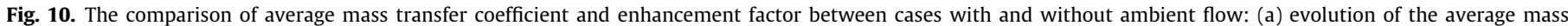
transfer coefficient during one cycle of the pendular activity, and (b) evolution of the enhancement factor.

confirming the local lengthening activity. The red spikes are also higher than the black ones indicating enhanced mass transfer and absorption. At $T / 2$, the strain rate is decreased to 0 (see
Fig. 6(a)), the blue spikes become even wider and higher (see Fig. 8(d)). After that, this section of wall starts to experience local shortening (see the negative strain rate in Fig. 6(a)). The flux distri- 
bution at $3 T / 4$ is almost identical to the one at $T / 4$ (Fig. 8(e) and (c)). At the end of one cycle, the flux distribution returns to the one at $t=0$ (Fig. 8(f)).

After introducing an ambient bulk flow, the absorption flux is increased dramatically. The peak value in Fig. 9 (a) is $2.07 \times 10^{-3}$ $\mathrm{mol} / \mathrm{m}^{2} \cdot \mathrm{s}$, which is more than 3.6 times higher than the peak value of $5.82 \times 10^{-4} \mathrm{~mol} / \mathrm{m}^{2} \cdot \mathrm{s}$ in Fig. 8(a). Another big difference between Fig. 9(a) and Fig. 8(a) is that, for the case with ambient bulk flow, absorption occurs throughout the whole duodenum. Concentration gradients can be observed above all villi in Fig. 7 (b), while it exists in the entrance region only in Fig. 7(a).

Taking a closer look at the section between 0 to $l$, a number of spikes with peaks and valleys can be identified as well (Fig. 9(b)). The peak value of flux decreases from $2.07 \times 10^{-3} \mathrm{~mol} / \mathrm{m}^{2} \cdot \mathrm{s}$ to around $0.52 \times 10^{-3} \mathrm{~mol} / \mathrm{m}^{2} \cdot \mathrm{s}$ within the section between $0 \mathrm{~mm}$ and $4.5 \mathrm{~mm}$ (i.e., $l$ ). Beyond $l$, the peak value of flux remains more or less the same. As shown in Fig. 9(c)-(f), similar to the case without ambient flow, the spikes become wider and higher during 0 to $T / 2$ implying local lengthening activity experienced by this section of wall. From $T / 2$ to $T$, local shortening movement offers thinner and shorter spikes.

It has been shown in Figs. 8 and 9 that the absorption flux and mass transfer coefficient is not uniformly distributed on the rough

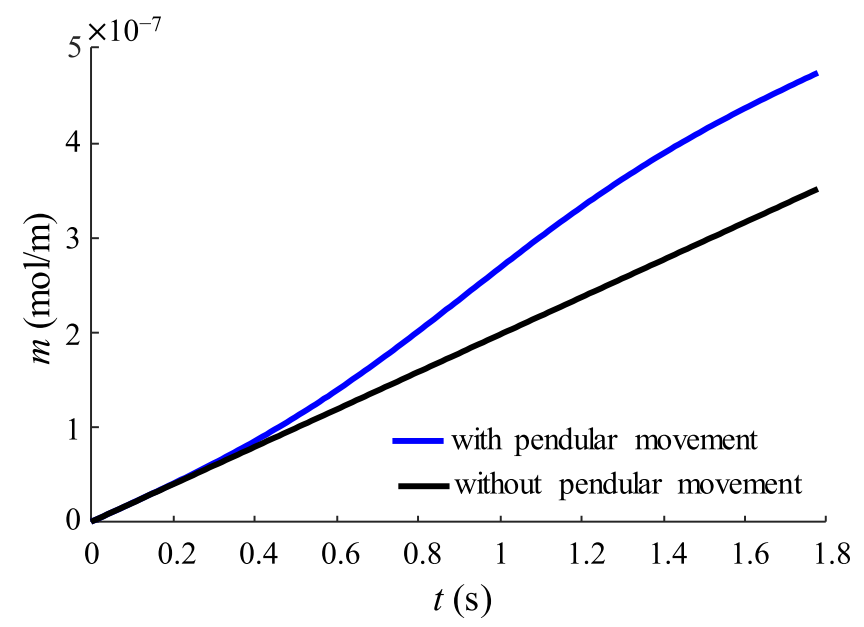

Fig. 11. Evolution of absorbed mass by the duodenum when there is no ambient bulk flow: comparison between the cases with and without pendular activity. absorption surface. The distribution is influenced by the structure of the villi. In this study, spikes with peaks and valleys have been observed. In order to see clearly the evolution of absorption flux or mass transfer coefficient during one cycle of the pendular activity, the mass transfer coefficient is averaged over the villi surface using Eq. (16). The average value is given in Fig. 10(a), where $\bar{K}$ corresponds to the case with pendular activity and $\bar{K}^{*}$ corresponds to the case without pendular activity. Regardless of whether there is an ambient bulk flow or not, mass transfer has been enhanced by the pendular activity. During one cycle of this activity, $\bar{K}$ increases from $K^{*}$ to a maximum value at $T / 2$, then it decreases back to around the original value at the end of the cycle. Introducing the ambient bulk flow leads to the significant increase of the mass transfer coefficient (see the blue lines well above the black lines in Fig. 10(a)). It is because axial transport of nutrients has been greatly enhanced by the ambient flow (see Fig. 7 and comparison between Fig. 8 and Fig. 9).

The enhancement of mass transfer due to pendular activity can be quantified using Eq. (17), which is a ratio between $\bar{K}$ and $\bar{K}^{*}$. The results in Fig. 10(b) shows that the maximum enhancement is achieved at $T / 2$ regardless of the ambient flow condition. For the case without ambient flow, the enhancement factor can reach as high as 1.72 , indicating a $72 \%$ increase of mass transfer due to the implementation of pendular activity. However, the maximum enhancement factor for the case with ambient flow is much lower, i.e., 1.1. Although the introduction of the ambient bulk flow can increase the mass transfer coefficient (see Fig. 10(a)) and hence the absorption flux (see comparison between Fig. 8(a) and Fig. 9 (a)), the enhancement of mass transfer due to the pendular activity is inhibited. It implies that pendular activity becomes less important for the enhancement of nutrient transfer and absorption when the ambient bulk flow is introduced into the system.

The amount of nutrients absorbed by the duodenum can be obtained using Eq. (19), which is given in Fig. 11. Without pendular activity, the mass increases linearly with time, indicating a constant absorption speed (i.e., the constant slope of the black curve in Fig. 11). The implementation of pendular activity can lead to the increase of absoprtion speed and hence the absorption mass. Two absorption speeds can be identified. The first and last quarters of the cycle have similar absorption speed as that of the case without pendular movement. However, from $T / 4$ to $3 T / 4$, the absorption speed is much higher (see the larger slope of the middle section of
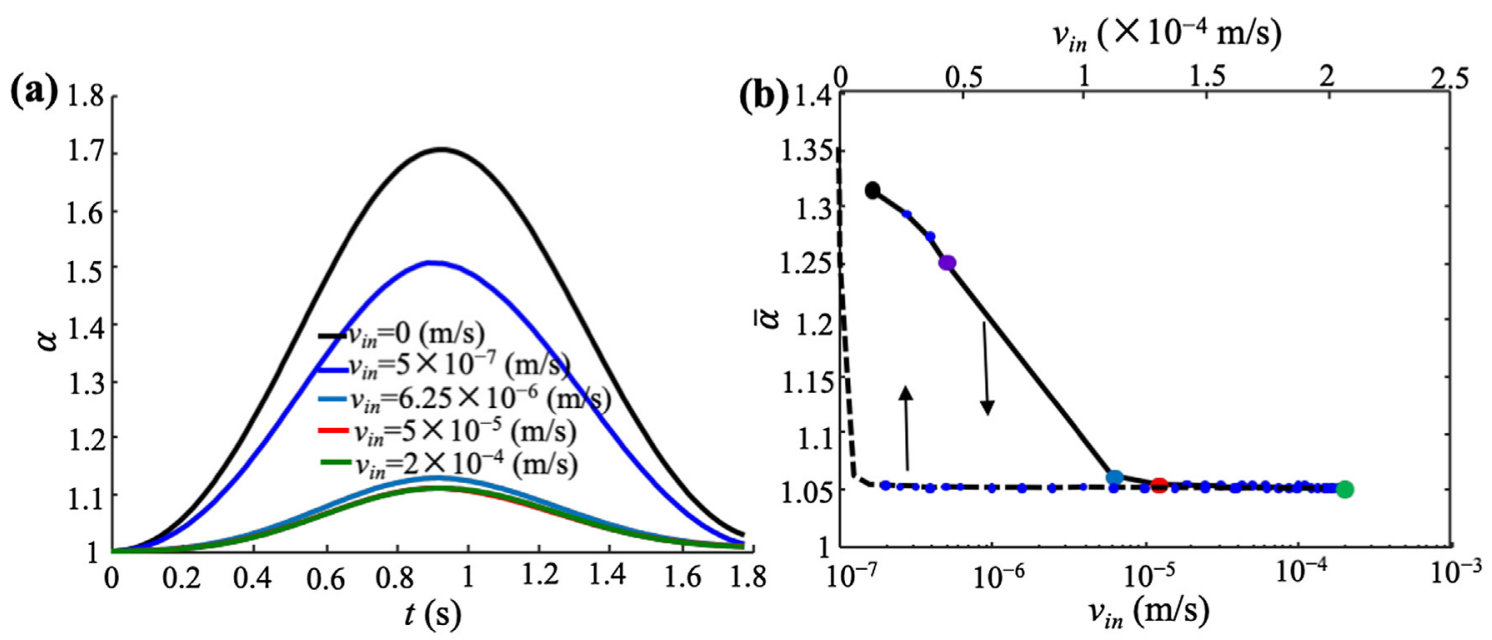

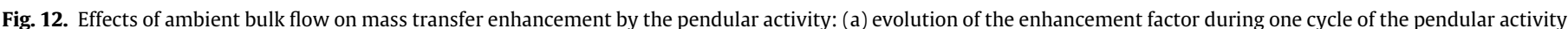

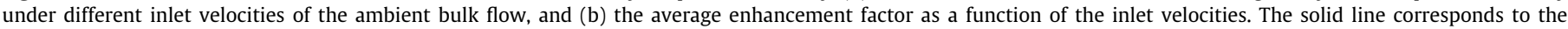
horizontal axis using the logarithmic scale and the dashed line corresponds to the horizontal axis using the linear scale. 
the blue curve in Fig. 11). This finding is consistent with analyses above on mass transfer coefficient and enhancement factor, where the maximum enhancement is achieved at $T / 2$ and the period from $T / 4$ to $3 T / 4$ is critical for enhanced mass absorption by the pendular activity (see Fig. 10).

\subsection{Effects of ambient bulk flow on mass transfer enhancement by pendular activity}

As discussed above, introducing ambient bulk flow will inhibit mass transfer enhancement by the pendular activity. A number of different inlet velocities have been tested. The evolution of the enhancement factor during one cycle of the pendular activity is plotted in Fig. 12 (a). The pattern of the curves under different inlet velocities is the same. The maximum enhancement is achieved at $T / 2$.

The average enhancement factor quantified by Eq. (18) is plotted as a function of the inlet velocity. When the inlet velocity increases slightly from $0 \mathrm{~m} / \mathrm{s}$ to $6.25 \times 10^{-6} \mathrm{~m} / \mathrm{s}$, the average enhancement factor decreases sharply from 1.35 to about 1.06 . Further increase of the inlet velocity to $5.0 \times 10^{-5} \mathrm{~m} / \mathrm{s}$ leads to a slight decrease of the average enhancement factor to 1.05 . For velocities above $5.0 \times 10^{-5} \mathrm{~m} / \mathrm{s}$ (up to $2 \times 10^{-4} \mathrm{~m} / \mathrm{s}$ ), the average enhancement factor maintains at 1.05 . It shows that at velocities higher than $5.0 \times 10^{-5} \mathrm{~m} / \mathrm{s}$, the mass transfer can be enhanced by $5 \%$ due to the pendular activity and this value is not affected by the inlet velocity. Although pendular activity becomes less important for the enhancement of nutrient transfer and absorption when the ambient bulk flow is introduced into the system, a 5\% enhancement can be ensured.

\section{Conclusion}

This work introduced a multi-physics model to quantitatively investigate how the motility can affect nutrient transfer and absorption in the duodenum. A unique feature of the current approach is that the movement of hierarchical structured lumen wall has been implemented using the moving mesh method. The structures of villi have been explicitly taken into account in the simulation.

It is found that the pendular activity can lead to regular vortex flow in the lumen. The absorption occurs mainly in the entrance region, i.e., the first vortex region. The mass transfer can be enhanced by the pendular activity and the maximum enhancement is achieved at $T / 2$. At $T / 2$, the maximum enhancement ratio is 1.72 , indicating that mass transfer and absorption can be enhanced by $72 \%$.

After introducing ambient bulk flow, axial transport of nutrients can be significantly improved. Absorption occurs over the entire duodenum. Thus the mass transfer coefficient and the amount of mass absorbed can be drastically increased as compared with the cases without ambient bulk flow. However, the mass transfer enhancement due to the pendular activity is inhibited. Slight increase of inlet velocity of bulk flow from $0 \mathrm{~m} / \mathrm{s}$ to $5.0 \times 10^{-5}$ $\mathrm{m} / \mathrm{s}$ can lead to drastic decrease of average enhancement ratio from 1.35 to 1.05 . Within the inlet velocity range investigated in this work $\left(0 \mathrm{~m} / \mathrm{s}\right.$ to $\left.2 \times 10^{-4} \mathrm{~m} / \mathrm{s}\right)$, a $5 \%$ enhancement of mass transfer due to pendular activity can be ensured.

Another interesting finding from the simulation results is that the absorption flux is distributed nonuniformly along the lumen wall. This distribution is affected by the villi structure. In this work, distribution curves of spikes with peaks and valleys are observed. The top of the villus demonstrates the highest absorption flux indicating its key role in nutrient absorption. The valley area, however, due to the high mass transfer resistance, has almost negligible absorption flux. A small scale 3D simulation of the first $4.5 \mathrm{~mm}$ section of the duodenum was carried out. Its' steady state solutions confirm the critical role in nutrient absorption played by the top part of the villi.

Although the specific system studied in this work is the rat duodenum, the modeling approach is generic and should be applicable to the investigation of the small intestine of other animals such as rabbits, pigs, and even humans. In a future work, the microvilli will be coupled into the current model system. Moreover, motility of the small intestine other than the pendular activity, such as segmentation and peristalsis, will be also taken into account. It has to be pointed out that the current geometry adopted for the $2 \mathrm{D}$ simulation cannot capture all features of a real 3D structure. If quantitatively-precise predictions are needed, modeling a complete 3D duodenum with all villi involved may be necessary in the future. In order to realize that objective, a lot of challenging issues have to be addressed, which include the implementation of the moving mesh approach for the motility of a complicated $3 \mathrm{D}$ structure as well as high computational complexity and memory demand. The results and conclusions offered by the current 2D model, however, have captured correctly the key trend of data and have already deepened our understanding of the mechanism of digestion, specifically in this study, how motility can enhance mass transfer and absorption in duodenum with villi explicitly considered. It should also be noted that the finding of enhanced mass transfer led by the well-coordinated local movement of the hierarchical-structured wall in this paper has the potential to inspire new ideas in the design of novel mixers or reactors, such as soft elastic reactors (Delaplace et al., 2018; Liu et al., 2018; Xiao et al., 2018).

\section{Author contributions}

Prof. Jie Xiao conceptualized the project, designed the simulation cases, developed analysis methods, analyzed the data and revised the manuscript. Ms. Yanan Zhang carried out all simulations, analyzed the data and drafted the manuscript. Prof. Xiao Dong Chen, Prof. Romain Jeantet, Dr. Didier Dupont, Dr. Guillaume Delaplace, and Dr. Peng Wu were all involved in data analysis and manuscript revision.

\section{Declaration of Competing Interest}

The authors declare that they have no known competing financial interests or personal relationships that could have appeared to influence the work reported in this paper.

\section{Acknowledgements}

This work was supported by the research funds from the National Natural Science Foundation of China (No. 21978184), the Natural Science Foundation of Jiangsu Province (Grant No. BK20170062), the National Key Research and Development Program of China (International S\&T Cooperation Program, ISTCP, No. 2016YFE0101200), the COFCO Nutrition and Health Research Institute (Beijing), and the "Jiangsu Specially-Appointed Professors Program", “Jiangsu Innovation and Entrepreneurship (Shuang Chuang) Program", "Priority Academic Program Development (PAPD) of Jiangsu Higher Education Institutions”.

\section{References}

Baklis, S., Tharakan, A., Jaimefonseca, M.R., Fryer, P.J., Norton, I.T., 2009. Modelling of physical and chemical processes in the small intestine. Royal Soc. Chem. 21, 377-383. 
Barry, R.E., 1976. Mucosal surface areas and villous morphology of the small intestine of small mammals: functional interpretations. J. Mammal. 57, 273290.

Bornhorst, G.M., Gouseti, O., Wickham, M.S., Bakalis, S., 2016. Engineering digestion: multiscale processes of food digestion. J. Food Sci. 81, R534-R543.

Canto, M.I., Setrakian, S., Petras, R.E., Blades, E., Chak, A., Sivak, M.V., 1996. Methylene blue selectively stains intestinal metaplasia in Barrett's esophagus. Gastrointest. Endosc. 44, 1-7.

Christensen, J., 1971. The controls of gastrointestinal movements: some old and new views. N. Engl. J. Med. 285, 85-98.

COMSOL Multiphysics v. 5.4. www.comsol.com. COMSOL AB, Stockholm, Sweden.

Delaplace, G., Gu, Y., Liu, M., Jeantet, R., Xiao, J., Chen, X.D., 2018. Homogenization of liquids inside a new soft elastic reactor: revealing mixing behavior through dimensional analysis. Chem. Eng. Sci. 192, 1071-1080.

De Loubens., C., Lentle, R.G., Love, R.J., Hulls, C., Janssen, P.W., 2013. Fluid mechanical consequences of pendular activity, segmentation and pyloric outflow in the proximal duodenum of the rat and the guinea pig. J. Royal Soc. Interface, vol. 10, 20130027-20130027.

Fonseca, M.R.J., 2012. An Engineering Understanding of the Small Intestine. University of Birmingham.

Gouseti, O., Jaime-Fonseca, M.R., Fryer, P.J., Mills, C., Wickham, M.S.J., Bakalis, S., 2014. Hydrocolloids in human digestion: dynamic in-vitro assessment of the effect of food formulation on mass transfer. Food Hydrocolloids 42, 378-385.

Grivel, M.L., Ruckebusch, Y., 1972. The propagation of segmental contractions along the small intestine. J. Physiol. 227, 611-625.

Guseinov, T.S., Guseinova, S.T., 2008. Effect of dehydration on morphogenesis of the Iymphatic network and immune structures in the small intestine. Bull. Exp. Biol. Med. 145, 755-757.

Gwynne, R.M., Thomas, E.A., Goh, S.M., SjVall, H., Bornstein, J.C., 2004. Segmentation induced by intraluminal fatty acid in isolated guinea-pig duodenum and jejunum. J. Physiol. 556, 557-569.

Holm, M., Powell, T., Casselbrant, A., Johansson, B., Fandriks, L., 2001. Dynamic involvement of the inducible type of nitric oxide synthase in acid-induced duodenal mucosal alkaline secretion in the rat. Dig. Dis. Sci. 46, 1765-1771.

Janssen, P.W.M., Lentle, R.G., 2013. Spatiotemporal Mapping Techniques for Quantifying Gut motility. New Advances in Gastrointestinal Motility Research. Springer, Dordrecht, pp. 219-241.

Janssen, P.W.M., Lentle, R.G., Asvarujanon, P., Chambers, P., Stafford, K.J., Hemar, Y., 2007. Characterization of flow and mixing regimes within the ileum of the brushtail possum using residence time distribution analysis with simultaneous spatio-temporal mapping. J. Physiol. 582, 1239-1248.

Jeffrey, B., Udaykumar, H.S., Schulze, K.S., 2003. Flow fields generated by peristaltic reflex in isolated guinea pig ileum: impact of contraction depth and shoulders. Am. J. Physiol.-Gastrointest. Liver Physiol. 285, G907-G918.

Lammers, W.J., Stephen, B., Arafat, K., Manefield, G.W., 1996. High resolution electrical mapping in the gastrointestinal system: initial results. Neurogastroenterol. Motility Official J. Eur. Gastroint. Motility Soc. 8, 207-216.

Leiper, J.B., 2015. Fate of ingested fluids: factors affecting gastric emptying and intestinal absorption of beverages in humans. Nutr. Rev. 73 (suppl 2), 57-72.

Lentle, R.G., 2018. Deconstructing the physical processes of digestion: reductionist approaches may provide greater understanding. Food Funct. 9 (8), 4069-4084.

Lentle, R.G., Janssen, P.W., Deloubens, C., Lim, Y.F., Hulls, C,. Chambers, P., 2013. Mucosal microfolds augment mixing at the wall of the distal ileum of the brushtail possum. Neurogastroenterol. Motility, vol. 25, 11, pp. 881-e700.

Lentle, R.G., Janssen, P.W.M., 2008. Physical characteristics of digesta and their influence on flow and mixing in the mammalian intestine: a review. J. Comp. Physiol. [B] 178 (6), 673-690.
Lentle, R.G., Janssen, P.W.M., Asvarujanon, P., Stafford, K.J., Hemar, Y., 2007. High definition mapping of circular and longitudinal motility in the terminal ileum of the brushtail possum Trichosurus vulpecula with watery and viscous perfusates. J. Comp. Physiol. B. 177, 543-556.

Lentle, R.G. Loubens, C.D., 2015. A review of mixing and propulsion of chyme in the small intestine: fresh insights from new methods. J. Comp. Physiol. B. 185, 369387.

Lentle, R.G., Loubens, C.D., Hulls, C., Janssen, P.W.M., Golding, M.D., Chambers, J.P. 2012. A comparison of the organization of longitudinal and circular contractions during pendular and segmental activity in the duodenum of the rat and guinea pig. Neurogastroenterol. Motil. 24 (7), 686-695.

Lim, I.Y.F. 2015. Factors influencing mixing and mass transfer in the small intestine: a thesis presented in partial fulfilment of the requirements for the degree of Doctor of Philosophy in Digestive Biomechanics (Physical Process of Digestion) at Massey University. New Zealand Massey University, Turitea.

Lim, Y.F., De, L.C., Love, R.J., Lentle, R.G., Janssen, P.W., 2015. Flow and mixing by small intestine villi. Food Funct. 6, 1787-1795.

Lim, Y.F., Lentle, R.G., Janssen, P.W., Williams, M.A., De, L.C., Mansel, B.W., Chambers, P. 2014. Determination of Villous Rigidity in the Distal Ileum of the Possum (Trichosurus vulpecula). PLoS ONE 9, (6) e100140.

Lim, Y.F., Williams, M.A.K., Lentle, R.G., Janssen, P.W.M., Mansel, B.W., Keen, S.A.J., Chambers, P., 2013. An exploration of the microrheological environment around the distal ileal villi and proximal colonic mucosa of the possum (Trichosurus vulpecula). J. R. Soc. Interface 10 (81), 20121008.

Liu, M., Xiao, J., Chen, X.D., 2018. A soft-elastic reactor inspired by the animal upper digestion tract. Chem. Eng. Technol. 41, 1051-1056.

Melville, J., Macagno, E., Christensen, J., 1975. Longitudinal contractions in the duodenum: their fluid-mechanical function. Am. J. Physiol. 228, 1887-1892.

Moxon, T.E., Gouseti, O., Bakalis, S., 2016. In silico modelling of mass transfer \& absorption in the human gut. J. Food Eng. 176, 110-120.

Riahi, D.N., Roy, R., 2011. Mathematical modeling of peristaltic flow of chyme in small intestine. Appl. Appl. Math. 6, 428-444.

Schulze-Delrieu, K., 1999. Visual parameters define the phase and the load of contractions in isolated guinea pig ileum. Am. J. Physiol. 276, 1417-1424.

Schulze, K.S., 2015. The imaging and modelling of the physical processes involved in digestion and absorption. Acta Physiol. 213, 394-405.

Stoll, B.R., Batycky, R.P., Leipold, H.R., Milstein, S., Edwards, D.A., 2000. A theory of molecular absorption from the small intestine. Chem. Eng. Sci. 55, 473-489.

Tharakan, A., Norton, I.T., Fryer, P.J., Bakalis, S., 2010. Mass transfer and nutrient absorption in a simulated model of small intestine. J. Food Sci. 75 (6), E339E346.

Wang, Y., Brasseur, J.G., 2017. Three-dimensional mechanisms of macro-to-microscale transport and absorption enhancement by gut villi motions. Phys. Rev. E 95, (6) 062412.

Wang, Y., Brasseur, J.G., Banco, G.G., Webb, A.G., Ailiani, A.C., Neuberger, T., 2010. A multiscale lattice Boltzmann model of macro-to micro-scale transport, with applications to gut function. Philos. Trans. Roy. Soc. A Math. Phys. Eng. Sci. 368, 2863-2880.

Wright, N.D., Kong, F., Williams, B.S., Fortner, L., 2016. A human duodenum model (HDM) to study transport and digestion of intestinal contents. J. Food Eng. 171, $129-136$.

Xiao, J., Zou, C., Liu, M., Zhang, G., Delaplace, G., Jeantet, R., Chen, X.D., 2018. Mixing in a soft-elastic reactor (SER) characterized using an RGB based image analysis method. Chem. Eng. Sci. 181, 272-285. 